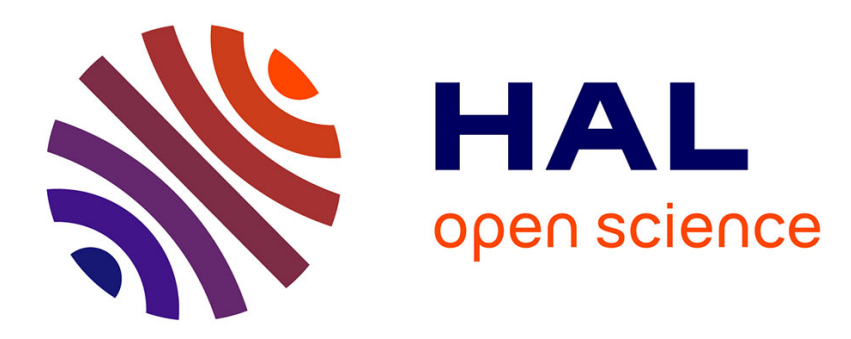

\title{
H-infinity unbiased filtering for linear descriptor systems via LMI
}

Mohamed Darouach

\section{To cite this version:}

Mohamed Darouach. H-infinity unbiased filtering for linear descriptor systems via LMI. IEEE Transactions on Automatic Control, 2009, 54 (8), pp.1966-1972. 10.1109/TAC.2009.2023962 . hal-00413701

\section{HAL Id: hal-00413701 \\ https://hal.science/hal-00413701}

Submitted on 4 Sep 2009

HAL is a multi-disciplinary open access archive for the deposit and dissemination of scientific research documents, whether they are published or not. The documents may come from teaching and research institutions in France or abroad, or from public or private research centers.
L'archive ouverte pluridisciplinaire HAL, est destinée au dépôt et à la diffusion de documents scientifiques de niveau recherche, publiés ou non, émanant des établissements d'enseignement et de recherche français ou étrangers, des laboratoires publics ou privés. 


\title{
$H_{\infty}$ unbiased filtering for linear descriptor systems via LMI
}

\author{
M. Darouach \\ Centre de Recherche en Automatique de Nancy (CRAN), Nancy-Université, CNRS, \\ 186 rue de Lorraine, 54400 Longwy, FRANCE \\ E-mails : Mohamed.Darouach@iut-longwy.uhp-nancy.fr
}

\begin{abstract}
This note concerns the optimal $H_{\infty}$ unbiased filtering problem for linear descriptor systems. It unifies the design of reduced-order, minimal-order and full-order filters for continuous and discrete-time systems. Necessary and sufficient conditions for the solvability of the problem are obtained in terms of a set of linear matrix inequalities (LMIs). The parametrization of all $H_{\infty}$ unbiased filters is presented. Application to standard systems with or without unknown inputs is given. A numerical example is given to show the applicability of the presented approach.
\end{abstract}

Keywords : Reduced order, Minimal order, Full order, Descriptor systems, LMI, Existence conditions, Unknown input estimation, Rectangular systems.

\section{Introduction and problem formulation}

Descriptor systems (known also as generalized, singular or differential algebraic (DA) systems) can describe a large class of systems, which are not only of theoretical interest but also have a great importance in practice. They are frequently encountered in chemical and mineral industries, in electronic and economic systems $[1,2]$. The state estimation problem for descriptor systems has been the subject of several studies in the past decades. We can distinguish two approaches, the Kalman filtering approach and $H_{\infty}$ approach. In the Kalman filtering, the system and the measurement noises are assumed to be Gaussian with known statistics [3], [4], [5]. When the noises are arbitrary signals with bounded energy, the $H_{\infty}$ filtering permits to guarantee a noise attenuation level [6].

Recently, a number of papers have appeared that deal with the $H_{\infty}$ filtering for descriptor systems, see for example [7], [8] and references therein. In all these works only full or reduced order filters were presented for the square descriptor systems. As one can see, the simultaneous state and unknown inputs estimation problems can be treated as a semi state estimation one for rectangular descriptor systems [9], where the semi state is formed by the state and the unknown inputs to be estimated. In our knowledge, the present work is the first one presenting in an unified framework the robust $H_{\infty}$ unbiased filtering for general rectangular descriptor systems. Reduced-order, minimal-order and full-order unbiased filters for continuous and discrete-time systems are presented in a compact formulation.

Consider the following descriptor system

$$
\begin{aligned}
& E \sigma x(t)=A x(t)+B u(t)+D_{1} w(t) \\
& y(t)=C x(t)+D_{2} w(t)
\end{aligned}
$$

with the initial semi state $x(0)=x_{0}$. Where $\sigma x(t)$ denotes the $\dot{x}(t)$ in the continuous case and $x(t+1)$ in the discrete case, $x(t) \in \mathbb{R}^{n}$ is the semi state vector, $u(t) \in \mathbb{R}^{m}$ is the known input, $w(t) \in \mathbb{R}^{n_{w}}$ is the disturbance vector containing both system and measurement noise, and $y(t) \in \mathbb{R}^{p}$ is the measurement output. matrix $E \in \mathbb{R}^{n_{1} \times n}$ and when $n_{1}=n$ matrix $E$ is singular with rank $E=r \leqslant n$. Matrices $A \in \mathbb{R}^{n_{1} \times n}, B \in \mathbb{R}^{n_{1} \times m}, C \in \mathbb{R}^{p \times n}, D_{1} \in \mathbb{R}^{n_{1} \times n_{w}}$ and $D_{2} \in \mathbb{R}^{p \times n_{w}}$ are real.

Let $\Phi \in \mathbb{R}^{r_{1} \times n_{1}}$ be a full row rank matrix such that $\Phi E=0$. In this case we have $r_{1}=n_{1}-r$, and from (1) we obtain $\Phi\left(A x(t)+D_{1} w(t)\right)=-\Phi B u(t)$.

In the sequel we assume that. 
Assumption 1. $\operatorname{rank}\left[\begin{array}{c}E \\ \Phi A \\ C\end{array}\right]=n$.

Remark 1. Assumption 1 is equivalent to the impulsive observability, i.e $\operatorname{rank}\left[\begin{array}{cc}E & A \\ 0 & C \\ 0 & E\end{array}\right]=n+\operatorname{rank} E$, in fact we have

$$
\begin{gathered}
\operatorname{rank}\left[\begin{array}{cc}
E & A \\
0 & C \\
0 & E
\end{array}\right]=\operatorname{rank}\left[\begin{array}{cc}
\Phi & 0 \\
E E^{+} & 0 \\
0 & I
\end{array}\right]\left[\begin{array}{cc}
E & A \\
0 & C \\
0 & E
\end{array}\right] \\
=\operatorname{rank}\left[\begin{array}{cc}
0 & \Phi A \\
E & E E^{+} A \\
0 & C \\
0 & E
\end{array}\right]=\operatorname{rank}\left[\begin{array}{cc}
0 & \Phi A \\
E & E E^{+} A \\
0 & C \\
0 & E
\end{array}\right]\left[\begin{array}{cc}
I & -E^{+} A \\
0 & I
\end{array}\right]=\operatorname{rank}\left[\begin{array}{c}
E \\
\Phi A \\
C
\end{array}\right]+\operatorname{rank} E .
\end{gathered}
$$

This condition is more general than the one ( $\operatorname{rank}\left[\begin{array}{l}E \\ C\end{array}\right]=n$ ) generally considered, see for example [10], [11].

Consider the following reduced-order filter for system (1).

$$
\begin{aligned}
& \sigma \zeta(t)=N \zeta(t)+J y(t)+H u(t) \\
& \widehat{x}(t)=P \zeta(t)-Q \Phi B u(t)+F y(t)
\end{aligned}
$$

Vector $\zeta(t) \in \mathbb{R}^{q}$ represents the state vector of the observer and $\widehat{x}(t) \in \mathbb{R}^{n}$ is the estimate of $x(t)$. Matrices $N, J, H, P, Q$, and $F$ are unknown matrices of appropriate dimensions, which must be determined.

Let $T \in \mathbb{R}^{q \times n_{E}}$ be a parameter matrix and define $\epsilon(t)=\zeta(t)-T E x(t)$, the error between $\zeta(t)$ and $T E x(t)$, then its dynamic is given by

$$
\begin{aligned}
\sigma \epsilon(t) & =\sigma \zeta(t)-T E \sigma x(t) \\
& =N \epsilon(t)+(N T E-T A+J C) x(t)+(H-T B) u(t)+\left(J D_{2}-T D_{1}\right) w(t)
\end{aligned}
$$

On the other hand from (2) and the definition of $\epsilon(t)$, we have

$$
\widehat{x}(t)=P \epsilon(t)+\left[\begin{array}{lll}
P & Q & F
\end{array}\right]\left[\begin{array}{c}
T E \\
\Phi A \\
C
\end{array}\right] x(t)+\left(Q \Phi D_{1}+F D_{2}\right) w(t)
$$

Now, if $\left[\begin{array}{lll}P & Q & F\end{array}\right]\left[\begin{array}{c}T E \\ \Phi A \\ C\end{array}\right]=I$ and by the unbiasedness of the filter for $w(t)=0$, the estimation error dynamic is independent of $x(t)$ and $u(t)$, we obtain:

$$
\begin{aligned}
& N T E-T A+J C=0 \\
& H=T B \\
& {\left[\begin{array}{lll}
P & Q & F
\end{array}\right]\left[\begin{array}{c}
T E \\
\Phi A \\
C
\end{array}\right]=I}
\end{aligned}
$$

In this case from (3) and (4) we obtain the following filtering error system

$$
\begin{array}{r}
\sigma \epsilon(t)=N \epsilon(t)+\mathbf{M} w(t) \\
e(t)=\widehat{x}(t)-x(t)=P \epsilon(t)+\mathbf{Q}_{F} w(t)
\end{array}
$$

where $\mathbf{M}=J D_{2}-T D_{1}$ and $\mathbf{Q}_{F}=Q \Phi D_{1}+F D_{2}$.

Now the problem of the unbiased optimal filter design is reduced to determine the matrices $N, J, H$, $P, Q, F$, and $T$ such that equations (5) are satisfied and the worst case filteringerror energy over all bounded energy disturbances $w(t)$ is minimized. 
Remark 2. The filter (2) is of dimension $q \leqslant n$ equal to the dimension of the matrix parameter $T$. When $q=n$ we obtain the full-order filter, for $q=n-p$ the obtained filter is of reduced-order one, and when $q=n-p-r_{1}$ the obtained filter is of minimal-order. When $n=p+r_{1}$, the filter is of order $q=0$, in this case the filter in (2) is reduced to the static filtering: $\widehat{x}(t)=-Q \Phi B u(t)+F y(t)$, with $\left[\begin{array}{ll}Q & F\end{array}\right]=\left[\begin{array}{c}\Phi A \\ C\end{array}\right]^{-1}$

\section{Optimal unbiased filtering}

Before giving the solution to the optimal filtering problem, we begin by solving the constrained Sylvester equations (5). Let $R \in \mathbb{R}^{q \times n}$ be any full row rank matrix such that $\Omega=\left[\begin{array}{c}R \\ \Phi A \\ C\end{array}\right]$ is of full column rank matrix. Define the following matrices: $\Gamma=\left[\begin{array}{c}E \\ \Phi A \\ C\end{array}\right], \Lambda_{1}=R \Gamma^{+}\left[\begin{array}{c}I_{n_{1}} \\ 0\end{array}\right], \Delta_{1}=\left(I_{n_{1}+r_{1}+p}-\Gamma \Gamma^{+}\right)\left[\begin{array}{c}I_{n_{1}} \\ 0\end{array}\right]$, $\Lambda_{2}=R \Gamma^{+}\left[\begin{array}{c}0 \\ I_{r_{1}+p}\end{array}\right], \Delta_{2}=\left(I_{n_{1}+r_{1}+p}-\Gamma \Gamma^{+}\right)\left[\begin{array}{c}0 \\ I_{r_{1}+p}\end{array}\right], \mathbf{A}_{1}=\Lambda_{1} A \Omega^{+}\left[\begin{array}{c}I_{q} \\ 0\end{array}\right], \mathbf{B}_{1}=\Delta_{1} A \Omega^{+}\left[\begin{array}{c}I_{q} \\ 0\end{array}\right], \mathbf{C}_{1}=$ $\left(I_{q+r_{1}+p}-\Omega \Omega^{+}\right)\left[\begin{array}{c}I_{q} \\ 0\end{array}\right], P_{1}=\Omega^{+}\left[\begin{array}{c}I_{q} \\ 0\end{array}\right]$, and $T^{\prime}=T-\Psi \Phi$, where $\Psi$ is an arbitrary matrix. The solution to (5) is given by the following lemma

Lemma 1. Under assumption 1, the general solution to (5) is given by

$$
\begin{gathered}
N=\mathbf{A}_{1}-Z_{1} \mathbf{B}_{1}-Y_{1} \mathbf{C}_{1} \\
T=T^{\prime}+\Psi \Phi \\
P=P_{1}-Y_{2} \mathbf{C}_{1}
\end{gathered}
$$

and

$$
\left[\begin{array}{cc}
-\Psi & J \\
Q & F
\end{array}\right]=\left(\left[\begin{array}{c}
T^{\prime} A \\
I_{n}
\end{array}\right] \Omega^{+}-\left[\begin{array}{c}
Y_{1} \\
Y_{2}
\end{array}\right]\left(I_{q+r_{1}+p}-\Omega \Omega^{+}\right)\right)\left[\begin{array}{c}
K \\
I_{r_{1}+p}
\end{array}\right]
$$

where $T^{\prime}=\Lambda_{1}-Z_{1} \Delta_{1}, K=\Lambda_{2}-Z_{1} \Delta_{2}$, with $Z_{1}, Y_{1}$ and $Y_{2}$ arbitrary matrices of appropriate dimensions. Proof. By using the definition of $T^{\prime}$ we can see that equations (5a) and (5c) can be written as

$$
\left[\begin{array}{ccc}
N & -\Psi & J \\
P & Q & F
\end{array}\right]\left[\begin{array}{c}
T^{\prime} E \\
\Phi A \\
C
\end{array}\right]=\left[\begin{array}{c}
T^{\prime} A \\
I_{n}
\end{array}\right]
$$

where we have used the fact that $\Phi E=0$.

Now the necessary and sufficient condition for (11) to have a solution is

$$
\operatorname{rank}\left[\begin{array}{c}
T^{\prime} E \\
\Phi A \\
C
\end{array}\right]=\operatorname{rank}\left[\begin{array}{c}
T^{\prime} E \\
\Phi A \\
C \\
T^{\prime} A \\
I_{n}
\end{array}\right]=n
$$

On the other hand, from the definition of matrix $\Omega$ and $\Gamma$, since $\operatorname{rank}\left[\begin{array}{l}\Gamma \\ R\end{array}\right]=\operatorname{rank} \Gamma$, condition (12) is equivalent to the existence of a parameter matrix $K$ such that

$$
T^{\prime} E+K\left[\begin{array}{c}
\Phi A \\
C
\end{array}\right]=\left[\begin{array}{ll}
T^{\prime} & K
\end{array}\right] \Gamma=R
$$


In this case the general solution to (13) is given by

$$
\begin{gathered}
T^{\prime}=\Lambda_{1}-Z_{1} \Delta_{1} \\
K=\Lambda_{2}-Z_{1} \Delta_{2}
\end{gathered}
$$

where $Z_{1}$ is an arbitrary matrix of appropriate dimension. Iserting (14) into (11) leads to

$$
\left[\begin{array}{ccc}
N & -\Psi & J \\
P & Q & F
\end{array}\right]\left[\begin{array}{cc}
I_{q} & -K \\
0 & I_{r_{1}+p}
\end{array}\right] \Omega=\left[\begin{array}{c}
T^{\prime} A \\
I_{n}
\end{array}\right]
$$

which has a solution, since $\Omega$ is of full column rank, given by

$$
\left[\begin{array}{ccc}
N & -\Psi & J \\
P & Q & F
\end{array}\right]=\left[\left[\begin{array}{c}
T^{\prime} A \\
I_{n}
\end{array}\right] \Omega^{+}-\left[\begin{array}{c}
Y_{1} \\
Y_{2}
\end{array}\right]\left(I_{q+r_{1}+p}-\Omega \Omega^{+}\right)\right]\left[\begin{array}{cc}
I_{q} & K \\
0 & I_{r_{1}+p}
\end{array}\right]
$$

where $Y_{1}$ and $Y_{2}$ are arbitrary matrices of appropriate dimensions.

Substituting the values of $T^{\prime}$ and $K$ in these equation leads to

$$
\begin{gathered}
N=\mathbf{A}_{1}-Z_{1} \mathbf{B}_{1}-Y_{1} \mathbf{C}_{1} \\
P=P_{1}-Y_{2} \mathbf{C}_{1}
\end{gathered}
$$

From (17) we also have

$$
\left[\begin{array}{cc}
-\Psi & J \\
Q & F
\end{array}\right]=\left[\left[\begin{array}{c}
T^{\prime} A \\
I_{n}
\end{array}\right] \Omega^{+}-\left[\begin{array}{c}
Y_{1} \\
Y_{2}
\end{array}\right]\left(I_{q+r_{1}+p}-\Omega \Omega^{+}\right)\right]\left[\begin{array}{c}
K \\
I_{r_{1}+p}
\end{array}\right]
$$

which proves the lemma.

Now, by inserting the obtained values in (20) into the expressions of $\mathbf{M}$ and $\mathbf{Q}_{F}$ we obtain $\mathbf{M}=$ $J D_{2}-T D_{1}=J D_{2}-T^{\prime} D_{1}-\Psi \Phi D_{1}=\left[\begin{array}{ll}-\Psi & J\end{array}\right]\left[\begin{array}{c}\Phi D_{1} \\ D_{2}\end{array}\right]-T^{\prime} D_{1}$ and $\mathbf{Q}_{F}=Q \Phi D_{1}+F D_{2}=\left[\begin{array}{ll}Q & F\end{array}\right]\left[\begin{array}{c}\Phi D_{1} \\ D_{2}\end{array}\right]$ Let $\bar{\Delta}_{2}=\Delta_{2}\left[\begin{array}{c}\Phi D_{1} \\ D_{2}\end{array}\right]$ and $Z_{1}=Z\left(I-\bar{\Delta}_{2} \bar{\Delta}_{2}^{+}\right)$, where $Z$ is an arbitrary matrix of appropriate dimension. Then we obtain the following expressions for $T^{\prime}, K, N$ and $\left[\begin{array}{cc}-\Psi & J \\ Q & F\end{array}\right]\left[\begin{array}{c}\Phi D_{1} \\ D_{2}\end{array}\right]$.

$$
\begin{gathered}
T^{\prime}=\Lambda_{1}-Z\left(I-\bar{\Delta}_{2} \bar{\Delta}_{2}^{+}\right) \Delta_{1} \\
K=\Lambda_{2}-Z\left(I-\bar{\Delta}_{2} \bar{\Delta}_{2}^{+}\right) \Delta_{2} \\
N=\mathbf{A}_{1}-Z \overline{\mathbf{B}}_{1}-Y_{1} \mathbf{C}_{1} \\
\left.\left[\begin{array}{cc}
-\Psi & J \\
Q & F
\end{array}\right]\left[\begin{array}{c}
\Phi D_{1} \\
D_{2}
\end{array}\right]=\left[\begin{array}{c}
T^{\prime} A \\
I_{n}
\end{array}\right] \Omega^{+}-\left[\begin{array}{c}
Y_{1} \\
Y_{2}
\end{array}\right]\left(I_{q+r_{1}+p}-\Omega \Omega^{+}\right)\right]\left[\begin{array}{c}
K \\
I_{r_{1}+p}
\end{array}\right]\left[\begin{array}{c}
\Phi D_{1} \\
D_{2}
\end{array}\right]
\end{gathered}
$$

with $\overline{\mathbf{B}}_{1}=\left(I-\bar{\Delta}_{2} \bar{\Delta}_{2}^{+}\right) \Delta_{1} A \Omega^{+}\left[\begin{array}{l}I \\ 0\end{array}\right]$.

These equations lead to the following values for $\mathbf{M}$ and $\mathbf{Q}_{F}: \mathbf{M}=\left[\begin{array}{ll}-\Psi & J\end{array}\right]\left[\begin{array}{c}\Phi D_{1} \\ D_{2}\end{array}\right]-T^{\prime} D_{1}=\alpha-$

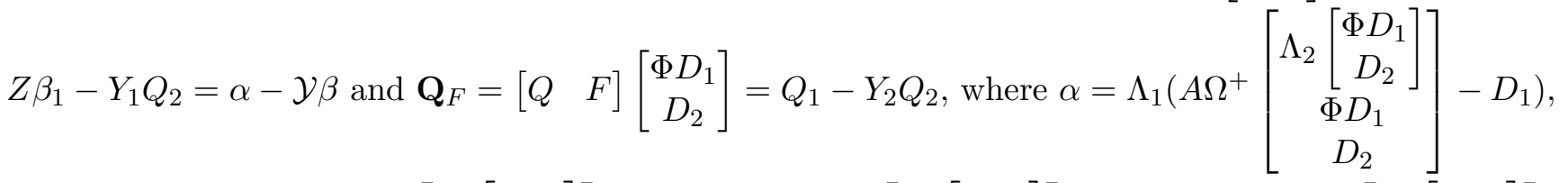

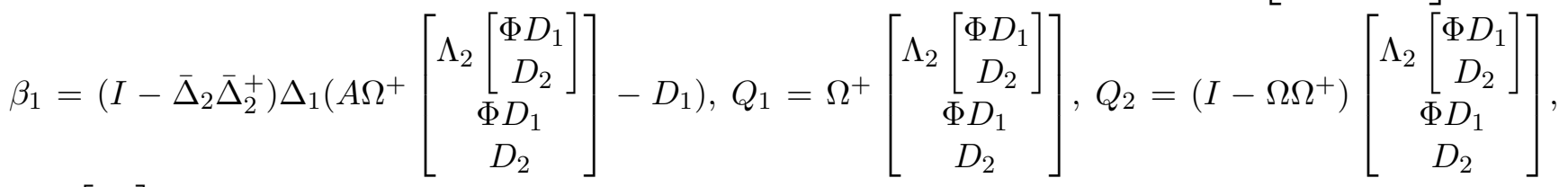
$\beta=\left[\begin{array}{l}\beta 1 \\ Q_{2}\end{array}\right]$, and $\mathcal{Y}=\left[\begin{array}{ll}Z & Y_{1}\end{array}\right]$ 
Now, let $\mathcal{B}=\left[\begin{array}{l}\overline{\mathbf{B}}_{1} \\ \mathbf{C}_{1}\end{array}\right]$, then equation (6) can be written as

$$
\begin{array}{r}
\sigma \epsilon(t)=\left(\mathbf{A}_{1}-\mathcal{Y} \mathcal{B}\right) \epsilon(t)+(\alpha-\mathcal{Y} \beta) w(t) \\
e(t)=\left(P_{1}-Y_{2} \mathbf{C}_{1}\right) \epsilon(t)+\left(Q_{1}-Y_{2} Q_{2}\right) w(t)
\end{array}
$$

Remark 3. When matrix $E$ is of full row rank we have $\Phi=0$ and the above results can be applied by taking $\Psi=0$ and $Q=0$. This case leads to the following filter

$$
\begin{aligned}
& \sigma \zeta(t)=N \zeta(t)+J y(t)+H u(t) \\
& \widehat{x}(t)=P \zeta(t)+F y(t)
\end{aligned}
$$

and the following matrices, $\Gamma=\left[\begin{array}{l}E \\ C\end{array}\right], \Omega=\left[\begin{array}{l}R \\ C\end{array}\right]$ are of full column rank matrices. Equation (24) becomes

$$
\left[\begin{array}{l}
J \\
F
\end{array}\right] D_{2}=\left[\left[\begin{array}{c}
T^{\prime} A \\
I_{n}
\end{array}\right] \Omega^{+}-\left[\begin{array}{c}
Y_{1} \\
Y_{2}
\end{array}\right]\left(I_{q+r_{1}+p}-\Omega \Omega^{+}\right)\right]\left[\begin{array}{c}
K \\
I_{r_{1}+p}
\end{array}\right] D_{2}
$$

In this case, matrices $\bar{\Delta}_{2}=\Delta_{2} D_{2}, T^{\prime}=T, \mathbf{M}=J D_{2}-T D_{1}=\alpha-Z \beta_{1}-Y_{1} Q_{2}=\alpha-\mathcal{Y} \beta, \mathbf{Q}_{F}=F D_{2}=$ $Q_{1}-Y_{2} Q_{2}$, where $\alpha=\Lambda_{1}\left(A \Omega^{+}\left[\begin{array}{c}\Lambda_{2} D_{2} \\ D_{2}\end{array}\right]-D_{1}\right), \beta_{1}=\left(I-\bar{\Delta}_{2} \bar{\Delta}_{2}^{+}\right) \Delta_{1}\left(A \Omega^{+}\left[\begin{array}{c}\Lambda_{2} D_{2} \\ D_{2}\end{array}\right]-D_{1}\right), Q_{1}=\Omega^{+}\left[\begin{array}{c}\Lambda_{2} D_{2} \\ D_{2}\end{array}\right]$, and $Q_{2}=\left(I-\Omega \Omega^{+}\right)\left[\begin{array}{c}\Lambda_{2} D_{2} \\ D_{2}\end{array}\right]$.

\subsection{Optimal $H_{\infty}$ filtering for the continuous time case}

In this section we present a method for designing an unbiased $\mathcal{H}_{\infty}$ filter for the continuous system described by (1), where $\sigma x(t)=d x / d t$. This problem is reduced to find the parameter matrices $\mathcal{Y}=$ $\left[\begin{array}{ll}Z & Y_{1}\end{array}\right]$ and $Y_{2}$ such that the worst estimation error energy $\|e\|_{\mathcal{L}_{2}}$ is minimum for all bounded energy disturbance $w(t)$, this is equivalent to find these parameter matrices such that min $\sup _{w \in \mathcal{L}_{2}-\{0\}} \frac{\|e\|_{\mathcal{L}_{2}}}{\|w\|_{\mathcal{L}_{2}}}$ is realized. This problem is equivalent to $\min \left\|T_{w e}\right\|_{\infty}$, where $T_{w e}$ is the transfer function from $w(t)$ to the estimation error $e(t)$. Here we shall design a $\gamma$-suboptimal filter such that $\left\|T_{w e}\right\|_{\infty}<\gamma$, where $\gamma$ is a given positive scalar. The solution to this problem is given by the following theorem.

Theorem 1. Under assumption 1, there exists a continuous unbiased filter (2) such that the filtering error system in (25) is stable and $\left\|T_{w e}\right\|_{\infty}<\gamma$, if and only if there exist a matrix $X=X^{T}>0$ and a matrix $Y_{2}$ satisfying the following LMIs.

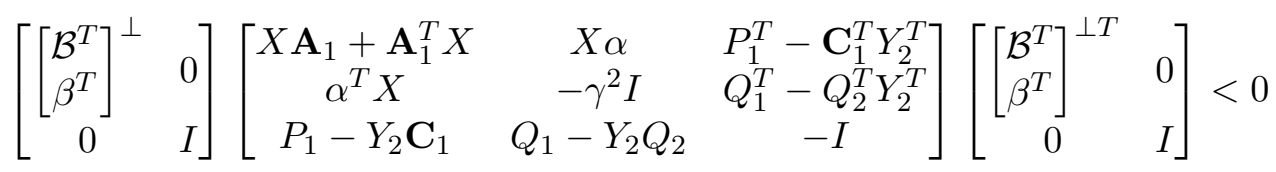

and

$$
\left[\begin{array}{cc}
-\gamma^{2} I & Q_{1}^{T}-Q_{2}^{T} Y_{2}^{T} \\
Q_{1}-Y_{2} Q_{2} & -I
\end{array}\right]<0
$$

In this case, all matrices $\mathcal{Y}$ are parametrized as follows

$$
\mathcal{Y}=\mathbb{G}_{R}^{+} \mathbb{K B}_{L}^{+}+\mathbb{Z}-\mathbb{G}_{R}^{+} \mathbb{G}_{R} \mathbb{Z B}_{L} \mathbb{B}_{L}^{+}
$$

where

$$
\begin{aligned}
\mathbb{K} & =-\mathbb{R}^{-1} \mathbb{G}_{L}^{T} \mathbb{S}_{1} \mathbb{B}_{R}^{T}\left(\mathbb{B}_{R} \mathbb{S}_{1} \mathbb{B}_{R}^{T}\right)^{-1}+\mathbb{R}^{-1} \mathbb{S}^{1 / 2} \mathbb{L}\left(\mathbb{B}_{R} \mathbb{S}_{1} \mathbb{B}_{R}^{T}\right)^{-1 / 2} \\
\mathbb{S}_{1} & =\left(\mathbb{G}_{L} \mathbb{R}^{-1} \mathbb{G}_{L}^{T}-\mathbb{Q}\right)^{-1}>0 \\
\mathbb{S} & =\mathbb{R}-\mathbb{G}_{L}^{T}\left(\mathbb{S}_{1}-\mathbb{S}_{1} \mathbb{B}_{R}^{T} \mathbb{B}_{R} \mathbb{S}_{1} \mathbb{B}_{R}^{T}\right) \mathbb{G}_{L}
\end{aligned}
$$


with $\mathbb{Q}=\left[\begin{array}{ccc}X \mathbf{A}_{1}+\mathbf{A}_{1}^{T} X & X \alpha & P_{1}^{T}-\mathbf{C}_{1}^{T} Y_{2}^{T} \\ \alpha^{T} X & -\gamma^{2} I & Q_{1}^{T}-Q_{2}^{T} Y_{2}^{T} \\ P_{1}-Y_{2} \mathbf{C}_{1} & Q_{1}-Y_{2} Q_{2} & -I\end{array}\right], \mathbb{B}=\left[\begin{array}{lll}\mathcal{B} & \beta & 0\end{array}\right], \mathbb{G}=\left[\begin{array}{c}-X \\ 0 \\ 0\end{array}\right]$, where $\mathbb{R}$ and $\mathbb{Z}$ are arbitrary matrices of appropriate dimensions satisfying $\mathbb{R}=\mathbb{R}^{T}>0$ and $\|\mathbb{L}\|<1$. Matrices $\mathbb{B}_{L}, \mathbb{B}_{R}, \mathbb{C}_{L}$ and $\mathbb{C}_{R}$ are any full rank matrices such that $\mathbb{B}=\mathbb{B}_{L} \mathbb{B}_{R}$ and $\mathbb{G}=\mathbb{G}_{L} \mathbb{G}_{R}$.

Proof. The bounded real lemma [12] guarantees that the filter error (25) is stable and the $\mathcal{H}_{\infty}$-norm bound $\left\|T_{w e}\right\|_{\infty}<\gamma$ if and only if there exists a matrix $X$ such that

$$
\left[\begin{array}{cc}
X N+N^{T} X+P^{T} P & X \mathbf{M}+P^{T} \mathbf{Q}_{F} \\
\mathbf{M}^{T} X+\mathbf{Q}_{F}^{T} P & -\gamma^{2} I+\mathbf{Q}_{F}^{T} \mathbf{Q}_{F}
\end{array}\right]<0
$$

Applying the Schur lemma we obtain

$$
\left[\begin{array}{ccc}
X N+N^{T} X & X \mathbf{M} & P^{T} \\
\mathbf{M}^{T} X & -\gamma^{2} I & \mathbf{Q}_{F}^{T} \\
P & \mathbf{Q}_{F} & -I
\end{array}\right]<0
$$

By inserting the values of $N, P, \mathbf{M}$ and $\mathbf{Q}_{F}$ in this inequality, we obtain

$$
\left[\begin{array}{ccc}
X \mathbf{A}_{1}+\mathbf{A}_{1}^{T} X-X \mathcal{Y B}-\mathcal{B}^{T} \mathcal{Y}^{T} X & X \alpha-X \mathcal{Y} \beta & P_{1}^{T}-\mathbf{C}_{1}^{T} Y_{2}^{T} \\
\alpha^{T} X-\beta^{T} \mathcal{Y}^{T} X & -\gamma^{2} I & Q_{1}^{T}-Q_{2}^{T} Y_{2}^{T} \\
P_{1}-Y_{2} \mathbf{C}_{1} & Q_{1}-Y_{2} Q_{2} & -I
\end{array}\right]<0
$$

which can be written as

$$
\mathbb{Q}+\mathbb{G} \mathcal{Y} \mathbb{B}+(\mathbb{G} \mathcal{Y} \mathbb{B})^{T}<0
$$

The solvability conditions of (35) are

$$
\begin{aligned}
\mathbb{G}^{\perp} \mathbb{Q} \mathbb{G}^{\perp T} & <0 \\
\mathbb{B}^{T \perp} \mathbb{Q B}^{T \perp T} & <0
\end{aligned}
$$

Condition (36a) is equivalent to $(29)$, since $\mathbb{G}^{\perp}=\left[\begin{array}{lll}0 & I & 0 \\ 0 & 0 & I\end{array}\right]$ and condition (36b) is exactly (28).

From [12], if these conditions are satisfied, all parameters $\mathcal{Y}$ that provide unbiased $\gamma$-suboptimal $\mathcal{H}_{\infty}$ filters are parametrized as in (30)-(31).

Remark 4. The parameter matrices $\mathcal{Y}$ and $Y_{2}$ can also be obtained as follows, equation (34) can be written as

$$
\left[\begin{array}{ccc}
X \mathbf{A}_{1}+\mathbf{A}_{1}^{T} X-\Omega_{\mathcal{Y}} \mathcal{B}-\mathcal{B}^{T} \Omega_{\mathcal{Y}}^{T} & X \alpha-\Omega_{\mathcal{Y}} \beta & P_{1}^{T}-\mathbf{C}_{1}^{T} Y_{2}^{T} \\
\alpha^{T} X-\beta^{T} \Omega_{\mathcal{Y}}^{T} & -\gamma^{2} I & Q_{1}^{T}-Q_{2}^{T} Y_{2}^{T} \\
P_{1}-Y_{2} \mathbf{C}_{1} & Q_{1}-Y_{2} Q_{2} & -I
\end{array}\right]<0
$$

where $\Omega_{\mathcal{Y}}=X \mathcal{Y}$. Then by computing the solution of the $L M I$ (37) with respect to $\Omega_{\mathcal{Y}}, Y_{2}$ and $X=$ $X^{T}>0$, we obtain the parameter matrix $\mathcal{Y}$ from $\mathcal{Y}=X^{-1} \Omega_{\mathcal{Y}}$.

\subsection{Optimal $H_{\infty}$ filtering for the discrete-time case}

In this section we present the unbiased $\mathcal{H}_{\infty}$ filter design for discrete-time systems described by (1), where $\sigma x(t)=x(t+1)$. From the above results, as in the continuous-time case this problem is reduced to find the parameter matrix $\mathcal{Y}$ solving min $\sup _{w \in l_{2}-\{0\}} \frac{\|e\|_{l^{2}}}{\|w\|_{l^{2}}}$. This problem is equivalent to min $\left\|T_{w e}\right\|_{\infty}$, where $T_{w e}$ is the transfer function from $w(t)$ to the estimation error $e(t)$. The solution to this problem is given by the following theorem 
Theorem 2. Under assumption 1, there exists a discrete-time unbiased filter (2) such that the discretetime filtering error system in (25) is stable and $\left\|T_{w e}\right\|_{\infty}<\gamma$ if and only if there exist a matrix $X=X^{T}>0$ and a matrix $Y_{2}$ such that

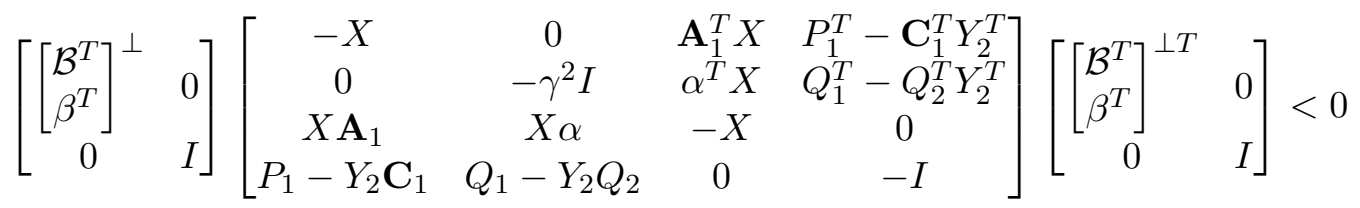

and

$$
\left[\begin{array}{ccc}
-I & \alpha^{T} & Q_{1}^{T}-Q_{2}^{T} Y_{2}^{T} \\
\alpha & -X & 0 \\
Q_{1}-Y_{2} Q_{2} & 0 & -\gamma^{2} I
\end{array}\right]<0
$$

In this case all the solution matrices $\mathcal{Y}$ are parametrized as follows

$$
\mathcal{Y}=\mathbb{S}_{1} \bar{\Gamma} R \Theta^{T} \mathbb{S} \bar{\Lambda}\left(\bar{\Lambda} \mathbb{S} \bar{\Lambda}^{T}\right)^{-1}+\varphi^{1 / 2} \mathbb{L}\left(\bar{\Lambda} \mathbb{S} \bar{\Lambda}^{T}\right)^{-1 / 2}
$$

where

$$
\begin{aligned}
\mathbb{S}_{1} & =\left(\bar{\Gamma}^{T} R \bar{\Gamma}\right)^{-1} \\
\mathbb{S} & =\left(Q-\Theta^{T} R \Theta+\Theta^{T} R \bar{\Lambda} \mathbb{S}_{1} \bar{\Lambda}^{T} R \Theta\right)^{-1} \\
\varphi & =\mathbb{S}_{1}-\mathbb{S}_{1} \bar{\Gamma}^{T} R \Theta\left(\mathbb{S}-\mathbb{S}_{1} \bar{\Lambda}^{T}\left(\bar{\Lambda} \mathbb{S} \bar{\Lambda}^{T}\right)^{-1} \bar{\Lambda} \mathbb{S}\right) \Theta^{T} R \bar{\Gamma} \mathbb{S}_{1}
\end{aligned}
$$

with $\Theta=\left[\begin{array}{cc}\mathbf{A}_{1} & \alpha \\ P_{1}-Y_{2} \mathbf{C}_{1} & Q_{1}-Y_{2} Q_{2}\end{array}\right], \bar{\Gamma}=\left[\begin{array}{c}-I \\ 0\end{array}\right], \bar{\Lambda}=\left[\begin{array}{ll}\mathcal{B} & \beta\end{array}\right], R=\left[\begin{array}{cc}X & 0 \\ 0 & I\end{array}\right], Q=\left[\begin{array}{cc}X & 0 \\ 0 & \gamma^{2} I\end{array}\right]$, and where $\mathbb{L}$ is an arbitrary matrix such that $\|\mathbb{L}\|<1$.

Proof. From the discrete-time bounded real lemma [12] the discrete-time filter error (25) is stable and $\left\|T_{w e}\right\|_{\infty}<\gamma$ if and only if there exists a matrix $X=X^{T}>0$ such that

$$
\left[\begin{array}{cc}
N & \mathbf{M} \\
P & \mathbf{Q}_{F}
\end{array}\right]^{T}\left[\begin{array}{cc}
X & 0 \\
0 & I
\end{array}\right]\left[\begin{array}{cc}
N & \mathbf{M} \\
P & \mathbf{Q}_{F}
\end{array}\right]<\left[\begin{array}{cc}
X & 0 \\
0 & \gamma^{2} I
\end{array}\right] .
$$

By inserting the values of $N, P, \mathbf{M}$ and $\mathbf{Q}_{F}$ in this inequality, we obtain the following inequality:

$$
(\Theta+\bar{\Gamma} \mathcal{Y} \bar{\Lambda})^{T} R(\Theta+\bar{\Gamma} \mathcal{Y} \bar{\Lambda})<Q
$$

The solvability conditions of (43) are

$$
\begin{aligned}
\bar{\Lambda}^{T \perp}\left(-Q+\Theta^{T} R \Theta\right) \bar{\Lambda}^{T \perp T} & <0 \\
\bar{\Gamma}^{\perp}\left(-R^{-1}+\Theta^{T} Q^{-1} \Theta\right) \bar{\Gamma}^{\perp T} & <0
\end{aligned}
$$

Now since $\bar{\Gamma}^{\perp}=\left[\begin{array}{ll}0 & I\end{array}\right]$, condition (44b) can be written as $-I+\left[\begin{array}{ll}\alpha^{T} & Q_{1}^{T}-Q_{2}^{T} Y_{2}^{T}\end{array}\right]\left[\begin{array}{cc}X^{-1} & 0 \\ 0 & \gamma^{-2} I\end{array}\right]\left[\begin{array}{c}\alpha \\ Q_{1}-Y_{2} Q_{2}\end{array}\right]<$ 0. By applying the schur complement to this LMI we obtain (39). On the other hand by using the Schur complement, condition (44a) can be written as $\left[\begin{array}{cc}\bar{\Lambda}^{T \perp} & 0 \\ 0 & I\end{array}\right]\left[\begin{array}{cc}-Q & \Theta^{T} R \\ R \Theta & -R\end{array}\right]\left[\begin{array}{cc}\bar{\Lambda}^{T \perp T} & 0 \\ 0 & I\end{array}\right]<0$, which is equivalent to (38). The parametrized solution can be obtained from [12].

Remark 5. The solution of the LMI (42) can also be obtained as follows. This LMI can be writen as

$$
\left[\begin{array}{cc|cc}
-X & 0 & N^{T} X & P^{T} \\
0 & -\gamma^{2} I & \mathbf{M}^{T} X & \mathbf{Q}_{F}^{T} \\
\hline X N & X \mathbf{M} & -X & 0 \\
P & \mathbf{Q}_{F} & 0 & -I
\end{array}\right]<0
$$


By inserting the values of $N, P, \mathbf{Q}_{F}$ and $\mathbf{M}$ in this inequality, we obtain

$$
\left[\begin{array}{cc|cc}
-X & 0 & \mathbf{A}_{1}^{T} X-\mathcal{B}^{T} \Omega_{\mathcal{Y}}^{T} & P_{1}^{T}-\mathbf{C}_{1}^{T} Y_{2}^{T} \\
0 & -\gamma^{2} I & \alpha^{T} X-\beta^{T} \Omega_{\mathcal{Y}}^{T} & Q_{1}^{T}-Q_{2}^{T} Y_{2}^{T} \\
\hline X \mathbf{A}_{1}-\Omega_{\mathcal{Y}} \mathcal{B} & X \alpha-\Omega \mathcal{Y} \beta & -X & 0 \\
P_{1}-Y_{2} \mathbf{C}_{1} & Q_{1}-Y_{2} Q_{2} & 0 & -I
\end{array}\right]<0
$$

where $\Omega_{\mathcal{Y}}=X \mathcal{Y}$. Then by computing the solution with respect to $\Omega \mathcal{Y}, Y_{2}$ and $X=X^{T}>0$, we obtain the parameter matrix $\mathcal{Y}$ from $\mathcal{Y}=X^{-1} \Omega \mathcal{Y}$.

Now we can summarize the presented approach in the following algorithm.

\section{Algorithm 1.}

1) Under assumption 1, find a matrix $R$ such that $\Omega=\left[\begin{array}{c}R \\ \Phi A \\ C\end{array}\right]$ is of full column rank, this can be done as follows, let $\left[\begin{array}{c}\Phi A \\ C\end{array}\right]=U\left[\begin{array}{cc}\Sigma & 0 \\ 0 & 0\end{array}\right] V^{T}$ be a singular value decomposition of $\left[\begin{array}{c}\Phi A \\ C\end{array}\right]$, where $U$ and $V$ are unitary matrices and $\Sigma$ is diagonal with positives entries, then we can choose the matrix $R=\left[\begin{array}{ll}I & 0\end{array}\right]\left[\begin{array}{cc}0 & I \\ \Sigma & 0 \\ 0 & 0\end{array}\right] V^{T}$, in this case we have $\left[\begin{array}{c}R \\ \Phi A \\ C\end{array}\right]=\left[\begin{array}{cc}I & 0 \\ 0 & U\end{array}\right]\left[\begin{array}{cc}0 & I \\ \Sigma & 0 \\ 0 & 0\end{array}\right] V^{T}$ which is of full column rank matrix. Then calculate $\Gamma, \Lambda_{1}, \Lambda_{2}, \mathbf{A}_{1}, Q_{1}, Q_{2}, \mathbf{C}_{1}, \overline{\mathbf{B}}_{1}, \Delta_{1}, \Delta_{2}, P_{1}, \alpha$, and $\beta$.

2) Solve the LMIs (28)-(29) for the continuous case, or the LMIs (38)-(39) for the discrete case, to obtain matrices $\mathcal{Y}$ and $Y_{2}$.

3) Compute matrices $T^{\prime}$ and $K$ given by (21)-(22). Then deduce the parameter matrices $\Psi, J, Q$ and $F$ from (20) and deduce $T=T^{\prime}+\Psi \Phi$. Then calculate the filter matrices $N$ given by (23), $H$ given by (5b) and $P$ given by (19).

\subsection{Particular cases}

In this section we show how the presented results can be used to design a full-order, reduced-order, and minimal-order filters for descriptor systems and for standard systems with or without unknown inputs.

\subsubsection{Descriptor systems}

\subsubsection{1 minimal order filter}

Let $\Phi \in \mathbb{R}^{r_{1} \times n_{1}}$ be a full row rank matrix such that $\Phi E=0$, with $\operatorname{rank} \Phi=r_{1}=n_{1}-r=\operatorname{rank} \Phi A$. Then the dimension of the minimal order observer is $q=n-r_{1}-p$, in this case matrix $\Omega=\left[\begin{array}{c}R \\ \Phi A \\ C\end{array}\right]$ is nonsingular and $\Omega^{+}=\Omega^{-1}=\left[\begin{array}{c}R \\ \Phi A \\ C\end{array}\right]^{-1}$. For this case, we obtain $\mathbf{C}_{1}=0, Q_{2}=0$ and from (19), (23) and (24) we have $P=P_{1}, N=\mathbf{A}_{1}-Z \overline{\mathbf{B}}_{1}$, and $\left[\begin{array}{cc}-\Psi & J \\ Q & F\end{array}\right]\left[\begin{array}{c}\Phi D_{1} \\ D_{2}\end{array}\right]=\left[\begin{array}{c}T^{\prime} A \\ I_{n}\end{array}\right] \Omega^{-1}\left[\begin{array}{c}K \\ I_{r_{1}+p}\end{array}\right]\left[\begin{array}{c}\Phi D_{1} \\ D_{2}\end{array}\right]$. It follows that $\mathbf{M}=\alpha-Z \beta_{1}$ and $\mathbf{Q}_{F}=Q_{1}$, then equation (25) can be written as

$$
\begin{aligned}
\sigma \epsilon(t) & =\left(\mathbf{A}_{1}-Z \overline{\mathbf{B}}_{1}\right) \epsilon(t)+\left(\alpha-Z \beta_{1}\right) w(t) \\
e(t) & =P_{1} \epsilon(t)+Q_{1} w(t)
\end{aligned}
$$

The design of the filter is reduced to the determination of the parameter matrix $Z$ and can be obtained from the solutions given in theorem 1 for the continuous case and from theorem 2 for the discrete-time 
case. The filter matrices can be obtained by following step 2 and step 3 of Algorithm 1 presented in the precedent section.

\subsubsection{Reduced-order observers}

This case corresponds to the full state estimation using a filter of order $n-p$. It can be obtained when $\Phi=0$, in this case assumption1 becomes rank $\left[\begin{array}{l}E \\ C\end{array}\right]=n$. It corresponds to $\Gamma=\left[\begin{array}{l}E \\ C\end{array}\right]$ and $\Omega=\left[\begin{array}{l}R \\ C\end{array}\right]$ nonsingular, then $\Omega^{+}=\Omega^{-1}$. The reduced-order filter design can be obtained as in the case of minimal order presented above in section a.

\subsubsection{Full order observers}

This case corresponds to the estimation of the full state by using a full-order filter. It corresponds to $q=n$ and matrix $R=I_{n}$, then we have, under assumption1, i.e $\operatorname{rank} \Gamma=n$, with $\Gamma=\left[\begin{array}{c}E \\ \Phi A \\ C\end{array}\right]$, $\Omega^{+}=\left[\begin{array}{c}R \\ \Phi A \\ C\end{array}\right]^{+}=\left[\begin{array}{ll}I_{n} & 0\end{array}\right], \mathbf{A}_{1}=\Lambda_{1} A, \mathbf{B}_{1}=\Delta_{1} A, \mathbf{C}_{1}=\left[\begin{array}{c}0 \\ \Phi A \\ C\end{array}\right]$, and $P=I_{n}$. Where $\Lambda_{1}=\Gamma^{+}\left[\begin{array}{c}I_{n_{1}} \\ 0\end{array}\right]$, $\Delta_{1}=-\left(I_{n_{1}+r_{1}+p}-\Gamma \Gamma^{+}\right)\left[\begin{array}{c}I_{n_{1}} \\ 0\end{array}\right], \Lambda_{2}=\Gamma^{+}\left[\begin{array}{c}0 \\ I_{r_{1}+p}\end{array}\right], \Delta_{2}=-\left(I_{n_{1}+r_{1}+p}-\Gamma \Gamma^{+}\right)\left[\begin{array}{c}0 \\ I_{r_{1}+p}\end{array}\right], \bar{\Delta}_{2}=\Delta_{2}\left[\begin{array}{c}\Phi D_{1} \\ D_{2}\end{array}\right]$, $\alpha=\Lambda_{1}\left(A \Lambda_{2}\left[\begin{array}{c}\Phi D_{1} \\ D_{2}\end{array}\right]-D_{1}\right), \beta_{1}=\left(I-\bar{\Delta}_{2} \bar{\Delta}_{2}^{+}\right) \Delta_{1}\left(A \Lambda_{2}\left[\begin{array}{c}\Phi D_{1} \\ D_{2}\end{array}\right]-D_{1}\right), Q_{1}=\Lambda_{2}\left[\begin{array}{c}\Phi D_{1} \\ D_{2}\end{array}\right], Q_{2}=\left[\begin{array}{c}0 \\ \Phi A \\ C\end{array}\right]\left[\begin{array}{c}\Phi D_{1} \\ D_{2}\end{array}\right]$.

As in the above cases, the full order design can be obtained from step 2 and step 3 of Algorithm 1.

\subsubsection{Standard systems}

This case corresponds to $E=I$, then we have $\Phi=0, \Gamma=\left[\begin{array}{l}I \\ C\end{array}\right], T^{\prime}=T$, and $\Omega=\left[\begin{array}{l}R \\ C\end{array}\right]$. In the sequel we shal present only the full order case, the reduced order case (which is also the minimal order) corresponds to $\Omega$ non singular and can be obtained from the minimal case presented for descriptor systems in the above section a.

\subsubsection{Full order filter}

This case corresponds to the dimension of the filter $q=n$. The value of matrix $R$ is $R=I_{n}$ and we have $\Gamma=\Omega=\left[\begin{array}{l}I \\ C\end{array}\right]$. It follows that $\Omega^{+}=\Gamma^{+}=\left[\begin{array}{ll}I & 0\end{array}\right]$. From the results of section II we have $\Lambda_{1}=I, \mathbf{A}_{1}=A$, $\Delta_{1}=\left[\begin{array}{c}0 \\ -C\end{array}\right], \Lambda_{2}=0, \Delta_{2}=\left[\begin{array}{l}0 \\ I\end{array}\right], \mathbf{C}_{1}=\left[\begin{array}{c}0 \\ -C\end{array}\right]=\Delta_{1}, \mathbf{B}_{1}=\Delta_{1} A=\left[\begin{array}{c}0 \\ -C A\end{array}\right], P=I_{n}, \bar{\Delta}_{2}=\Delta_{2}\left[\begin{array}{c}0 \\ D_{2}\end{array}\right]$, $\bar{\Delta}_{2}^{+}=\left[\begin{array}{ll}0 & D_{2}^{+}\end{array}\right], \overline{\mathbf{B}}_{1}=\left[\begin{array}{c}0 \\ -\left(I-D_{2} D_{2}^{+}\right) C A\end{array}\right], \alpha=-D_{1}, \beta_{1}=\left[\begin{array}{c}0 \\ -\left(I-D_{2} D_{2}^{+}\right) C D_{1}\end{array}\right], Q_{1}=0, Q_{2}=\left[\begin{array}{c}0 \\ D_{2}\end{array}\right]$.

Now, let $Z=\left[\begin{array}{ll}Z_{1} & Z_{2}\end{array}\right], Y_{1}=\left[\begin{array}{ll}Y_{11} & Y_{12}\end{array}\right]$ and $Y_{2}=\left[\begin{array}{ll}Y_{21} & Y_{22}\end{array}\right]$ be the partitions of matrices $Z, Y_{1}$ and $Y_{2}$ respectively according to the partitions of $\overline{\mathbf{B}}_{1}, Q_{2}$, and $\beta_{1}$ then we have $P=P_{1}-Y_{2} \mathbf{C}_{1}=I+Y_{22} C$, $\left.\mathbf{Q}_{F}=Q_{1}-Y_{2} Q_{2}=-Y_{22} D_{2}, \mathbf{M}=\alpha-\mathcal{Y} \beta=-D_{1}-Z_{2}\left(I-D_{2} D_{2}^{+}\right) C D_{1}-Y_{12} D_{2}, \mathbf{A}_{1}-\mathcal{Y} \mathcal{B}\right)=$ $A+Z_{2}\left(I-D_{2} D_{2}^{+}\right) C A+Y_{12} C, T^{\prime}=T=I+Z_{2}\left(I-D_{2} D_{2}^{+}\right) C$, and $K=-Z_{2}\left(I-D_{2} D_{2}^{+}\right)$. Let $\overline{\mathcal{B}}=\left[\begin{array}{c}-\left(I-D_{2} D_{2}^{+}\right) C A \\ -C\end{array}\right], \bar{\beta}=\left[\begin{array}{c}\left(I-D_{2} D_{2}^{+}\right) C D_{1} \\ D_{2}\end{array}\right]$ and $\mathcal{Y}_{1}=\left[\begin{array}{ll}Z_{2} & Y_{12}\end{array}\right]$, then equation (6) becomes

$$
\begin{aligned}
\sigma \epsilon(t) & =\left(A-\mathcal{Y}_{1} \overline{\mathcal{B}}\right) \epsilon(t)+\left(-D_{1}-\mathcal{Y}_{1} \bar{\beta}\right) w(t) \\
e(t) & =\left(I+Y_{22} C\right) \epsilon(t)-Y_{22} D_{2} w(t)
\end{aligned}
$$

The design of the filter is reduced to the determination of the parameter matrices $Z_{2}, Y_{22}$ and $Y_{12}$. The filter matrices be obtained by following step 2 and step 3 of Algorithm 1 presented in the precedent section. 
Remark 6. From the above results, for $Y_{22}=0$ and $Z_{2}=0$, the filter (2) becomes

$$
\sigma \widehat{x}(t)=\left(A+Y_{12} C\right) \widehat{x}(t)-Y_{12} y(t)+B u(t)
$$

and the dynamic error (6) becomes

$$
\sigma e(t)=\left(A+Y_{12} C\right) e(t)+\left(-D_{1}-Y_{12} D_{2}\right) w(t)
$$

Now, let us consider the results of [6] for the full-order unbiased filtering, with the notations of [6] for $L=I$, the filter can be written as

$$
\sigma \widehat{x}(t)=H \widehat{x}(t)+J y(t)
$$

with $H=A-J C$ and $J=Z$, and the dynamic error

$$
\sigma e(t)=(A-Z C) e(t)+(B+Z D) w(t)
$$

with $e(t)=x(t)-\widehat{x}(t)$ which corresponds to our equations (47) and (48), where $e(t)=\widehat{x}(t)-x(t)$. In this case the LMIs (28)-(29) and (38)-(39) become exactly those obtained by [6] for $L=I$.

\subsubsection{State and unknown inputs estimation}

For simplicity we only present the standard discrete-time case, where the unknown inputs affects only the state equation, the general case where the unknown inputs are present in the measurements can be studied directly from this case. Let us consider the following discrete time systems with unknwon inputs

$$
\begin{aligned}
x(t+1) & =\bar{A} x(t)+B u(t)+\bar{F} d(t)+D_{1} w(t) \\
y(t) & =\bar{C} x(t)+D_{2} w(t)
\end{aligned}
$$

Where $x(t) \in \mathbb{R}^{n}$ is the state vector, $u(t) \in \mathbb{R}^{m}$ is the vector of the known inputs, $d(t) \in \mathbb{R}^{d}$ is the vector of the unknown inputs and $w(t) \in \mathbb{R}^{n_{w}}$ represents the disturbance vector. Matrices $\bar{A}, B, C, D_{1}$ and $D_{1}$ are of appropriate dimensions and $\bar{F} \in \mathbb{R}^{n \times d}$, without lost of generality we assume that $\bar{F}$ is of full column rank, i.e rank $\bar{F}=d$. This system can be written in a singular system form as

$$
\begin{aligned}
E X(t+1) & =A X(t)+B u(t)+D_{1} w(t) \\
y(t) & =C X(t)+D_{2} w(t)
\end{aligned}
$$

where $E=\left[\begin{array}{ll}I & -\bar{F}\end{array}\right], X(t)=\left[\begin{array}{c}x(t) \\ d(t-1)\end{array}\right], A=\left[\begin{array}{ll}\bar{A} & 0\end{array}\right], C=\left[\begin{array}{ll}\bar{C} & 0\end{array}\right]$.

Now, since $E$ is of full row rank see Remark 3, matrix $\Phi=0$ and $\Gamma$ reduces to $\Gamma=\left[\begin{array}{l}E \\ C\end{array}\right]$, the condition $\operatorname{rank} \Gamma=(n+d)$ is equivalent to $\operatorname{rank} \bar{C} \bar{F}=\operatorname{rank} \bar{F}$, which is the condition generally adopted for the unknown inputs observers, see [13] for example. This can be seen from rank $\left[\begin{array}{l}E \\ C\end{array}\right]=n+d=$ $\operatorname{rank}\left[\begin{array}{cc}I & -\bar{F} \\ \bar{C} & 0\end{array}\right]$. We shall consider the full-order case, i.e $q=n+d$, the reduced-order case $q=n+d-p$ can be obtained by using the results presented in section C-1. First, one can see that $R=I_{n+d}, \Gamma^{+}=$ $\left[\begin{array}{cc}I-\bar{F}(\bar{C} \bar{F})^{+} \bar{C} & \bar{F}(\bar{C} \bar{F})^{+} \\ -(\bar{C} \bar{F})^{+} \bar{C} & (\bar{C} \bar{F})^{+}\end{array}\right], \Omega=\left[\begin{array}{c}I_{n+d} \\ C\end{array}\right], \Omega^{+}=\left[\begin{array}{ll}I_{n+d} & 0\end{array}\right], \Lambda_{1}=\left[\begin{array}{c}I-\bar{F}(\bar{C} \bar{F})^{+} \bar{C} \\ -(\bar{C} \bar{F})^{+} \bar{C}\end{array}\right], \Lambda_{2}=\left[\begin{array}{c}\bar{F}(\bar{C} \bar{F})^{+} \\ (\bar{C} \bar{F})^{+}\end{array}\right]$ $\Delta_{1}=\left[\begin{array}{c}0 \\ -\left(\bar{C}-\bar{C} \bar{F}(\bar{C} \bar{F})^{+} \bar{C}\right)\end{array}\right], \Delta_{2}=\left[\begin{array}{c}0 \\ \left.I-\bar{C} \bar{F}(\bar{C} \bar{F})^{+}\right)\end{array}\right], \mathbf{A}_{1}=\Lambda_{1} A, \mathbf{C}_{1}=\left[\begin{array}{c}0 \\ -C\end{array}\right], P_{1}=I, \bar{\Delta}_{2}=\Delta_{2} D_{2}$, $\mathbf{Q}_{F}=F D_{2}, \overline{\mathbf{B}}_{1}=\left(I-\bar{\Delta}_{2} \bar{\Delta}_{2}^{+}\right) \Delta_{1} A, \alpha=\Lambda_{1}\left(A \Lambda_{2} D_{2}-D_{1}\right), Q_{1}=\Lambda_{2} D_{2}, Q_{2}=\left[\begin{array}{c}0 \\ D_{2}-C \Lambda_{2} D_{2}\end{array}\right], \beta_{1}=$ $\left(I-\bar{\Delta}_{2} \bar{\Delta}_{2}^{+}\right) \Delta_{1}\left(A \Lambda_{2} D_{2}-D_{1}\right), \beta=\left[\begin{array}{l}\beta_{1} \\ Q_{2}\end{array}\right]$, and $\mathcal{B}=\left[\begin{array}{l}\overline{\mathbf{B}}_{1} \\ \mathbf{C}_{1}\end{array}\right]$. The matrices of the filter can be obtained from step 2 and step 3 of Algorithm 1. 


\section{$3 \quad$ Numerical example}

This example concerns a square descriptor system presented in [7] and described by system (1) where $E=\left[\begin{array}{ccc}-2 & -2 & 0 \\ -2 & 4 & -6 \\ 1 & 4 & -3\end{array}\right], A=\left[\begin{array}{ccc}1.5 & 0.4 & -0.9 \\ -0.6 & -4.4 & 1.8 \\ 0.7 & 2.4 & 4.3\end{array}\right], D_{1}=\left[\begin{array}{cc}-0.6 & -1.1 \\ 4.2 & 5.2 \\ 5.2 & 3.7\end{array}\right], C=\left[\begin{array}{ccc}-0.4 & 1.2 & -0.4 \\ 1.7 & 1.4 & 0.7\end{array}\right], D_{2}=$ $\left[\begin{array}{cc}-1 & 0 \\ -1 & 0.5\end{array}\right]$. The problem here is to design a filter in the form (2) for the estimation of the full state. Here we have $\operatorname{rank} E=2$, in this case matrices $\phi=\left[\begin{array}{lll}-2 & 1 & -2\end{array}\right]$, and $\Phi A=\left[\begin{array}{lll}-5 & -10 & -5\end{array}\right]$. It is easy to see that, in this case the minimal order is $q=n-p-r_{1}=0$, it means that we can estimate de full state only from the measurements and the static equation described by $\Phi A$, see Remark 2 , in this case we obtain $\gamma$ optimal given by $\gamma=\left\|\mathbf{Q}_{F}\right\|=\sigma_{\max }\left(\mathbf{Q}_{F}\right)=3.784$ where $\mathbf{Q}_{F}=\left[\begin{array}{c}\Phi A \\ C\end{array}\right]^{-1}\left[\begin{array}{c}\Phi D_{1} \\ D_{2}\end{array}\right]$. Now for $q=1$, let $R=\left[\begin{array}{lll}1 & 0 & 0\end{array}\right]$ and take $\Phi=0$, in this case it is easy to see that $\Omega$ is nonsingular and $\Gamma=\left[\begin{array}{l}E \\ C\end{array}\right]$ is of full column rank. We obtain the following results, $X=199.50, Z=\left[\begin{array}{lll}6331 & 25324 & 6331\end{array}\right], T=$ $\left[\begin{array}{ll}8.4784-4.4180 & 8.9261\end{array}\right], K=\left[\begin{array}{ll}-0.8082 & -0.0755\end{array}\right]$. The obtained filter matrices are $N=-33.368$, $J=\left[\begin{array}{ll}26.2943 & 34.7054\end{array}\right], F=\left[\begin{array}{cc}-0.8082 & -0.0755 \\ 0.7309 & 0.3073 \\ 0.5009 & 0.9974\end{array}\right]$ and $P=\left[\begin{array}{c}1.0000 \\ -0.2857 \\ -1.8571\end{array}\right]$. The optimal $\mathcal{H}_{\infty}$ norm error is $\gamma=0.999$. When the order $q=2$, we obtain for the optimal $\mathcal{H}_{\infty}$ norm error $\gamma=0.6575$.

\section{Conclusion}

In this note, we have presented the $\mathcal{H}_{\infty}$ unbiased filtering for linear descriptor systems. The obtained results unify the filtering design of full, reduced and minimal orders for continuous and discrete-time systems. Necessary and sufficient conditions for the existence of these filters have been derived in terms of a set of LMIs. The parametrization of all $\mathcal{H}_{\infty}$ unbiased filters has been given.

\section{References}

[1] D. Luenberger, "Dynamic equations in descriptor form," IEEE Transactions on Automatic Contol., vol. 32 , pp. 312-321, 1977.

[2] L. Dai, Singular Control Systems, vol. 118 of Lecture Notes in Control and Information Sciences. New York: Springer-Verlag, 1989.

[3] M. Darouach, M. Zasadzinski, and D. Mehdi, "State estimation of stochastic singular linear systems," International Journal of Systems Science, vol. 24, pp. 345-354, 1993.

[4] R. Nikoukhah, A. Willsky, and B. Levy, "Kalman filtering and Riccati equations for descriptor systems," IEEE Transactions on Automatic Contol., vol. 37, pp. 1325-1342, 1992.

[5] R. Nikoukhah, S. Campbell, and F. Delebecque, "Kalman filtering for general discrete-time systems," IEEE Transactions on Automatic Contol., vol. 44, pp. 1829-1839, 1999.

[6] J. Watson and K. Grigoriadis, "Optimal unbiased filtering via linear matrix inequalities," Systems \& Control Letters, vol. 35, pp. 111-118, 1998.

[7] S. Xu, J.Lam, and Y.Zou, " $\mathcal{H}_{\infty}$ filtering for singular systems," IEEE Transactions on Automatic Contol., vol. 48, pp. 2217-2222, 2003.

[8] S. Xu and J.Lam, "Reduced-order $\mathcal{H}_{\infty}$ filtering for singular systems," Systems 8 Control Letters, vol. 56 , pp. $48-57,2007$. 
[9] M. Darouach, M. Zasadzinski, A. Bassong Onana, and S. Nowakowski, "Kalman filtering with unknown inputs via optimal state estimation of singular systems," International Journal of Systems Science, vol. 26, pp. 2015-2028, 1995.

[10] M. Darouach and M.Boutayeb, "Design of observers for descriptor systems," IEEE Transactions on Automatic Contol., vol. 40, pp. 1323-1327, 1995.

[11] M. Darouach, M.Zasadzinski, and M.Hayar, "Reduced-order observer design for descriptor systems with unknown inputs," IEEE Transactions on Automatic Contol., vol. 41, pp. 1068-1072, 1996.

[12] R. Skelton, T. Iwasaki, and K. Grigoriadis, A Unified Algebraic Approach to Linear Control Design. London: Taylor \& Francis, 1998.

[13] M. Darouach, M.Zasadzinski, and S.Xu, "Full-order observers for linear systems with unknown inputs," IEEE Transactions on Automatic Contol., vol. 39, pp. 606-609, 1994. 